PROCEEDINGS OF THE

AMERICAN MATHEMATICAL SOCIETY

Volume 133, Number 1, Pages 31-41

S 0002-9939(04)07549-5

Article electronically published on July 26, 2004

\title{
FINITE $s$-ARC TRANSITIVE CAYLEY GRAPHS AND FLAG-TRANSITIVE PROJECTIVE PLANES
}

\author{
CAI HENG LI
}

(Communicated by John R. Stembridge)

\begin{abstract}
In this paper, a characterisation is given of finite $s$-arc transitive Cayley graphs with $s \geq 2$. In particular, it is shown that, for any given integer $k$ with $k \geq 3$ and $k \neq 7$, there exists a finite set (maybe empty) of $s$-transitive Cayley graphs with $s \in\{3,4,5,7\}$ such that all $s$-transitive Cayley graphs of valency $k$ are their normal covers. This indicates that $s$-arc transitive Cayley graphs with $s \geq 3$ are very rare. However, it is proved that there exist 4arc transitive Cayley graphs for each admissible valency (a prime power plus one). It is then shown that the existence of a flag-transitive non-Desarguesian projective plane is equivalent to the existence of a very special arc transitive normal Cayley graph of a dihedral group.
\end{abstract}

\section{INTRODUCTION}

A graph $\Gamma$ is a Cayley graph if there exist a group $G$ and a subset $S \subset G$ with $S=S^{-1}:=\left\{s^{-1} \mid s \in S\right\}$ such that the vertices of $\Gamma$ may be identified with the elements of $G$ in such a way that $x$ is connected to $y$ if and only if $y x^{-1} \in S$. The Cayley graph $\Gamma$ is denoted by $\operatorname{Cay}(G, S)$. Cayley graphs stem from a type of diagram now called a Cayley color diagram, introduced by Cayley in 1878. In this paper, we investigate the symmetric Cayley graphs and a relation between them and finite flag-transitive projective planes.

A graph $\Gamma$ is said to be $(X, s)$-arc transitive if $X \leq A u t \Gamma$ is transitive on vertices and $s$-arcs of $\Gamma$ where $s$ is a positive integer. (A sequence $v_{0}, v_{1}, \ldots, v_{s}$ of vertices of $\Gamma$ is called an $s$-arc if $v_{i}$ is adjacent to $v_{i+1}$ for $0 \leq i \leq s-1$ and $v_{i-1} \neq v_{i+1}$ for $1 \leq i \leq s-1$.) An $(X, s)$-arc transitive graph is called $(X, s)$-transitive if it is not $(X, s+1)$-arc transitive. In particular, if $X=A u t \Gamma$, then an $(X, s)$-arc transitive graph and an $(X, s)$-transitive graph are simply called $s$-arc transitive and $s$-transitive, respectively.

Interest in $s$-arc transitive graphs stems from a seminal result of Tutte in 1947, who proved that there exist no finite $s$-transitive cubic graphs for $s \geq 6$. Tutte's Theorem was generalized by Weiss 24] who proved that there exist no finite $s$ transitive graphs of valency at least 3 for $s=6$ and $s \geq 8$. Since then, characterizing

Received by the editors August 27, 2003 and, in revised form, September 11, 2003 and September 24, 2003.

2000 Mathematics Subject Classification. Primary 20B15, 20B30, 05C25.

This work was supported by an Australian Research Council Discovery Grant, and a QEII Fellowship. The author is grateful to the referee for his constructive comments. 
$s$-arc transitive graphs has received considerable attention in the literature (see, for example, 11, 14, 19, 24]).

Let $G$ be a group, and let $\Gamma=\operatorname{Cay}(G, S)$. Then $\Gamma$ has an automorphism group:

$$
\hat{G}=\{\hat{g}: x \rightarrow x g \text { for all } x \in G \mid g \in G\},
$$

consisting of right multiplications of elements $g \in G$. The subgroup $\hat{G}$ acts regularly on the vertex set of $\Gamma$. If $\hat{G}$ is normal in Aut $\Gamma$, then $\Gamma$ is called a normal Cayley graph; if the core of $\hat{G}$ in Aut $\Gamma$ has index 2 in $\hat{G}$, then $\Gamma$ is called a bi-normal Cayley graph, while if $\hat{G}$ is core free in $\mathrm{Aut} \Gamma$, then $\Gamma$ is called a core free Cayley graph. (Recall that the core of a subgroup $H$ of a group $X$ is the largest normal subgroup of $X$ contained in $H$.) These three classes of Cayley graphs are important for studying $s$-arc transitive Cayley graphs. Normal Cayley graphs have some very nice properties; refer to 21, 25, and also to Lemma 2.2 and Proposition 2.3. Several nice properties of bi-normal Cayley graphs are given in Lemma 2.4 and Corollary 2.7 Some properties for core-free Cayley graphs are given in Section 3 An interesting result of Section 3 is Proposition 3.2, which says that

"almost all" vertex primitive Cayley graphs are normal Cayley graphs.

Let $N \triangleleft X$, and let $\mathcal{B}$ be the set of $N$-orbits in $V$. Then the normal quotient graph $\Gamma_{N}$ of $\Gamma$ induced by $N$ is the graph with vertex set $\mathcal{B}$ such that $B, B^{\prime} \in \mathcal{B}$ are adjacent if and only if some vertex $u \in B$ is adjacent in $\Gamma$ to some vertex $v \in B^{\prime}$. If $\Gamma$ and $\Gamma_{N}$ have the same valency, then $\Gamma$ is called a normal cover of $\Gamma_{N}$.

Some special classes of 2-arc transitive Cayley graphs have been studied; see [1. 2 11, 15, 18. One of the main results of this paper is stated in Theorem 1.1, which tells us that $s$-arc transitive Cayley graphs with $s \geq 3$ are rare. Throughout this paper, denote by $\mathcal{G}(s, k)$ the set of core-free $s$-transitive Cayley graphs of valency $k$.

Theorem 1.1. For any integers $s \in\{2,3,4,5,7\}$ and $k \geq 3$, the set $\mathcal{G}(s, k)$ is finite, and for each s-transitive Cayley graph $\Gamma$, one of the following statements holds:

(i) $\Gamma$ is a normal or a bi-normal Cayley graph, and either $s=2$, or $(s, k)=$ $(3,7)$ and $\Gamma$ is bi-normal (so bipartite);

(ii) $\Gamma$ is a normal cover of a member of $\mathcal{G}(s, k)$.

Moreover, $\mathcal{G}(2, k)$ and $\mathcal{G}(3, k)$ are not empty for all $k \geq 3$, and $\mathcal{G}(4, q+1)$ is not empty for all prime powers $q$; in particular, there exist 4-transitive Cayley graphs of all admissible valencies.

Remarks. (a) It is easily shown that complete graphs are members of $\mathcal{G}(2, k)$, and complete bipartite graphs are members of $\mathcal{G}(3, k)$. Some other examples of members in $\mathcal{G}(2, k)$ and $\mathcal{G}(3, k)$ will be constructed in Section 4 .

(b) The examples in $\mathcal{G}(4, q+1)$ with $q$ being a power of a prime are the incidence graphs of Desarguesian projective planes. It is known that the valency of a 4transitive graph is $r+1$ such that $r$ is a power of a prime; see [24. Thus, Theorem 1.1 tells us that for all admissible valencies, there exist 4-transitive Cayley graphs. However, we do not know any other examples of 4-transitive Cayley graphs, and we do not have any examples of 5- or 7-transitive Cayley graphs at all.

(c) There have been some results regarding certain special classes of 2-arc transitive normal and bi-normal Cayley graphs; see [2, 11, 15, 20]. However, the general 
case is still not quite well-understood. In particular, we do not know any examples of 3-transitive bi-normal Cayley graphs.

We would like to propose the following question and problem.

Question 1.2. (a) Do there exist 3-transitive bi-normal Cayley graphs?

(b) Do there exist $s$-transitive Cayley graphs for $s=5$ and $s=7$ ?

Problem 1.3. (a) Determine members of $\mathcal{G}(s, k)$ for $k \geq 3$ and $s \geq 2$.

(b) Give a satisfactory description of bi-normal 2-transitive Cayley graphs.

Let $\Pi$ be a projective plane of order $n$ with point set $\mathcal{P}$ and line set $\mathcal{L}$. Then $\Pi$ has $n^{2}+n+1$ points and $n^{2}+n+1$ lines such that (a) any two points lie on exactly one line and any two lines intersect at exactly one point, (b) each line contains exactly $n+1$ points and each point lies on exactly $n+1$ lines. A flag of $\Pi$ is a pair of point $\mathrm{p}$ and line $\mathrm{I}$ such that $\mathrm{p}$ lies on I. A permutation of flags preserving the incidence of $\Pi$ is an automorphism of $\Pi$, and all automorphisms of $\Pi$ form a group Aut $\Pi$; that is, the automorphism group of $\Pi$. A plane $\Pi$ is called flag-transitive if Aut $\Pi$ is transitive on its flags. Let $\Gamma$ be the incidence graph of $\Pi$; that is, the vertex set of $\Gamma$ is $\mathcal{P} \cup \mathcal{L}$, and the edge set of $\Gamma$ is the set of flags of $\Pi$. Then Aut $\Gamma=\operatorname{Aut} \Pi$ or $(\operatorname{Aut} \Pi) \cdot \mathbb{Z}_{2}$; so if $\Pi$ is flag-transitive, then $\Gamma$ is edge-transitive.

The classical (Desarguesian) projective planes have been well studied, and their incidence graphs give the first family of 4-arc transitive Cayley graphs, presented in Example 4.5. The existence problem of non-Desarguesian flag-transitive projective planes is a long-standing open problem in finite geometry; see, for example, books [ $\underline{6}$. 17, and articles [7, 12, 22. Here we prove that the existence of a non-Desarguesian projective plane is equivalent to the existence of a type of Cayley graph of a dihedral group satisfying very restricted properties.

Let $n$ be a 2-power such that $p:=n^{2}+n+1$ and $q:=n+1$ are primes. Let $G=\langle a\rangle \rtimes\langle z\rangle \cong \mathrm{D}_{2 p}$, where $a^{z}=a^{-1}$. Let $\sigma \in \operatorname{Aut}(\langle a\rangle)$ be such that $\sigma z=z \sigma$ and $o(\sigma z)=2 q$. Let

$$
S=(a z)^{\langle\sigma\rangle}=\left\{a z, a^{r} z, a^{r^{2}} z, \ldots, a^{r^{q-1}} z\right\},
$$

and set $\Gamma(q)=\operatorname{Cay}(G, S)$. It will be shown in Lemma 5.1 that $\Gamma(q)$ has full automorphism group $\operatorname{Aut} \Gamma(q)=G \rtimes\langle\sigma z\rangle \cong \mathbb{Z}_{p} \rtimes \mathbb{Z}_{2 q}$, a Frobenius group of order $2 p q$, and thus $\Gamma(q)$ is a normal Cayley graph of the dihedral group $G \cong \mathrm{D}_{2 p}$. The following theorem shows that the girth of $\Gamma(q)$ determines the existence of flagtransitive non-Desarguesian projective planes of order $q-1$.

Theorem 1.4. Using the notation defined above, there exists a flag-transitive nonDesarguesian projective plane of order $n$ if and only if the girth of the Cayley graph $\Gamma(q)$ is equal to 6.

As widely believed, all flag-transitive projective planes are Desarguesian, and hence we make the following conjecture; see Lemma [5.1.

Conjecture 1.5. For each prime power $q$, the graph $\Gamma(q)$ defined above has diameter 4 and girth 4 .

Theorem 1.4 tells us that if this conjecture is true, then there exist no finite non-Desarguesian flag-transitive projective planes. 


\section{Automorphism groups of CAyley graphs: Normality}

It is known that a graph $\Gamma$ is a Cayley graph of a group $G$ if and only if its automorphism group contains a subgroup that is isomorphic to $G$ and acts regularly on vertices; see, for example, [3. Proposition 16.3]. It is hence natural to use the regular subgroup to describe properties of $\Gamma$ and Aut $\Gamma$.

2.1. Normal Cayley graphs. Let $G$ be a finite group, and let $\Gamma=\operatorname{Cay}(G, S)$. Let

$$
\operatorname{Aut}(G, S)=\left\{\sigma \in \operatorname{Aut}(G) \mid S^{\sigma}=S\right\} .
$$

Let $\mathbb{1}$ be the vertex of $\Gamma$ corresponding to the identity of $G$. It follows from the definition that each element of $\operatorname{Aut}(G, S)$ induces an automorphism of $\Gamma$ fixing $\mathbb{1}$, and so $\operatorname{Aut}(G, S) \leq(\operatorname{Aut} \Gamma)_{\mathbb{1}}$. Moreover, we have the following statement.

Lemma $2.1([8])$. For a Cayley graph $\Gamma=\operatorname{Cay}(G, S)$, the following holds:

$$
\mathrm{N}_{\text {Aut } \Gamma}(\hat{G})=\hat{G} \rtimes \operatorname{Aut}(G, S) .
$$

The subgroup Aut $(G, S)$ plays an important role in the study of Cayley graphs; refer to $8,21,25$. Some structural information may be read out from $\operatorname{Aut}(G, S)$; for instance, if $\operatorname{Aut}(G, S)$ is transitive on $S$, then the Cayley graph $\Gamma$ is $\mathrm{N}_{\mathrm{Aut} \Gamma}(\hat{G})$-arc transitive; if $\operatorname{Aut}(G, S)$ is 2-transitive on $S$, then $\Gamma$ is $\left(\mathrm{N}_{\mathrm{Aut} \Gamma}(\hat{G}), 2\right)$-arc transitive.

For a group $X$ with $\hat{G} \leq X \leq \operatorname{Aut} \Gamma$, if $\hat{G}$ is normal in $X$, then $X_{\mathbb{1}} \leq \operatorname{Aut}(G, S)$, and $\Gamma$ is called an $X$-normal Cayley graph of $G$. In particular, if $X=A u t \Gamma$, then $\Gamma$ is a normal Cayley graph, and $\operatorname{Aut} \Gamma=\hat{G} \rtimes \operatorname{Aut}(G, S)$. The action of $X$ on the vertices of $\Gamma$ behaves in a very nice way:

Lemma 2.2. Let $\Gamma=\operatorname{Cay}(G, S)$, and let $X \leq \operatorname{Aut} \Gamma$ be such that $\hat{G}$ is a normal subgroup of $X$. Then $X=\hat{G} \rtimes X_{\mathbb{1}}$, and for an element $x=\hat{g} y \in X$, where $\hat{g} \in \hat{G}$ and $y \in X_{\mathbb{1}}$, and for any vertex $h \in G$, we have

$$
h^{x}=h^{\hat{g} y}=(h g)^{y}=y^{-1} h g y .
$$

This property implies the following result about $s$-arc transitive Cayley graphs, which was obtained in [13].

Proposition 2.3. Let $\Gamma=\operatorname{Cay}(G, S)$, and let $X \leq \operatorname{Aut} \Gamma$ be such that $\hat{G}$ is a normal subgroup of $X$. Then $\Gamma$ is not $(X, 3)$-arc transitive.

2.2. Bi-normal Cayley graphs. Let $\Gamma=\operatorname{Cay}(G, S)$, and let $\hat{G} \leq X \leq \operatorname{Aut} \Gamma$. In this section we study the important case where $\hat{G}$ is not normal in $X$ but core $_{X}(\hat{G})$ is "big". A Cayley graph $\operatorname{Cay}(G, S)$ is called an $X$-bi-normal Cayley graph of $G$ if the core core $X(\hat{G})$ has index 2 in $\hat{G}$. Hence if $X=A u t \Gamma$, then an $X$-bi-normal Cayley graph is a bi-normal Cayley graph.

For an $X$-normal Cayley graph $\Gamma=\operatorname{Cay}(G, S), X_{\mathbb{1}}$ acts on the vertex set $G$ by conjugation, and so if $\Gamma$ is connected, then $X_{\mathbb{1}}$ is faithful on $S$. For an $X$-bi-normal Cayley graph Cay $(G, S)$, the $X_{\mathbb{1}}$-action only on half of its vertices is by conjugation; however, $X_{\mathbb{1}}$ is still faithful on $S$ under certain conditions, as shown below.

Lemma 2.4. Let $\Gamma$ be a connected bipartite graph with biparts $U$ and $U^{\prime}$. Assume that $X \leq \mathrm{Aut} \Gamma$ is transitive on the vertex set $V \Gamma$, and assume furthermore that $X$ has a normal subgroup $N \leq X^{+}$such that $N$ is intransitive on $V \Gamma, N_{v}$ is transitive on $\Gamma(v)$ for some vertex $v \in U$, and $N$ has a normal subgroup that is regular on both $U$ and $U^{\prime}$. Then $N_{v}$ acts faithfully on $\Gamma(v)$. 
Proof. Choose $v \in U$, and let $R=M N_{v}^{[1]}$. Since $U$ is an orbit of $N$ and $M$ acts transitively on $U$, we have $N=M N_{v}$. Since $M$ is normal in $N$ and $N_{v}^{[1]}$ is normal in $N_{v}$, it follows that $R$ is normal in $N$ and that $R=M N_{w}^{[1]}$ (and hence $R_{w} \leq N_{w}^{[1]}$ ) for all $w \in U$. Now let $\{u, v\}$ be an arbitrary edge. We may assume that $u \in U$ and $v \in U^{\prime}$. Let $x \in R_{u}$. Since $R=M N_{v}^{[1]}$, we have $x=y z$ where $y \in M$ and $z \in N_{v}^{[1]}$. Since $N_{v}^{[1]} \leq R_{u}$, we have $z \in R_{u}$. It then follows that $y \in M \cap R_{u}$. By the assumption that $M$ is regular on $U$, we have $M \cap R_{u}=1$. Thus $y=1$. We conclude that $x=z \in N_{v}^{[1]}$, and so $R_{u} \leq N_{v}^{[1]} \leq R_{v}$. Since $\{u, v\}$ is arbitrary, it follows that $R_{u} \leq N_{u}^{[1]}$ for every $u \in U^{\prime}$. Thus $R_{u} \leq N_{u}^{[1]}$ for every $u \in V \Gamma$. Since $\Gamma$ is connected, it follows that $R_{u v}=1$ for every edge $\{u, v\}$. Therefore, $N_{u}^{[1]}=1$ for every vertex $u \in V \Gamma$.

By inspecting the classification of finite 2-transitive permutation groups (see [4]), the statement of the next lemma is easily obtained.

Lemma 2.5. Let $T$ be a 2-transitive permutation group on a set $\Omega$ of degree $n$. Then for two points $\omega, \omega^{\prime} \in \Omega$, the point stabilizer $T_{\omega \omega^{\prime}}$ has a transitive permutation representation of degree $n-1$ if and only if $\operatorname{soc}(T)=\mathrm{A}_{7}$ or $\mathrm{S}_{7}$ and $n=7$.

The next result tells us that for a $(G, s)$-arc transitive graph, if the vertex stabilizer is faithful on the neighborhood, then $s$ is small.

Proposition 2.6. Let $\Gamma$ be an $(X, s)$-transitive graph of valency $k$, where $G \leq \operatorname{Aut} \Gamma$ and $s \geq 1$. Assume that $X_{v}$ is faithful on $\Gamma(v)$. Then either $s \leq 2$, or $(k, s)=(7,3)$ and $X_{v} \cong \mathrm{A}_{7}$ or $\mathrm{S}_{7}$.

Proof. By Lemma 2.4 the vertex stabilizer $X_{v}$ acts faithfully on $\Gamma(v)$; that is, $X_{v} \cong X_{v}^{\Gamma(v)}$, and thus $X_{v}$ is a 2-transitive permutation group on $\Gamma(v)$. Suppose furthermore that $\Gamma$ is $(X, 3)$-arc transitive. Then for distinct vertices $u, w \in \Gamma(v)$, the stabilizer $X_{u v w}$ of the 2 -arc $(u, v, w)$ is transitive on $\Gamma(w) \backslash\{v\}$. In particular, $X_{u v w}$ has a transitive permutation representation of degree $k-1$. Note that $X_{u v w}$ is the stabilizer of $u, w$ of the 2-transitive permutation group $X_{v} \cong X_{v}^{\Gamma(v)}$. Thus by Lemma 2.5. we conclude that $X_{v} \cong \mathrm{A}_{7}$ or $\mathrm{S}_{7}, X_{v w} \cong \mathrm{A}_{6}$ of $\mathrm{S}_{6}$, and $X_{u v w} \cong \mathrm{A}_{5}$ or $\mathrm{S}_{5}$, respectively. In particular, $k=7$, completing the proof of the proposition.

Combining Lemma 2.4 and Proposition 2.6, we have the following statement for bi-normal Cayley graphs.

Corollary 2.7. Let $\Gamma=\operatorname{Cay}(G, S)$ be a connected graph of valency $k$. Assume that $\hat{G}<X \leq$ Aut $\Gamma$ is such that $\Gamma$ is $X$-bi-normal and $(X, 2)$-arc transitive. Then $X_{\mathbb{1}}$ is faithful on $\Gamma(\mathbb{1})$, and furthermore, either

(i) $\Gamma$ is not $(X, 3)$-arc transitive, or

(ii) $k=7, X_{\mathbb{1}} \cong \mathrm{A}_{7}$ or $\mathrm{S}_{7}$, and $\Gamma$ is $(X, 3)$-transitive.

\section{Core-Free CAYley graphs and their group DUALS}

Let $\Gamma=\operatorname{Cay}(G, S)$, and let $X \leq \operatorname{Aut} \Gamma$ contain $\hat{G}$. Assume furthermore that $\hat{G}$ is not normal in $X$. If the core of $\hat{G}$ in $X$ is trivial, then $\Gamma$ is a core-free Cayley graph for if $\hat{G}$ is core-free in $X$, then $\hat{G}$ is core-free in $\mathrm{Aut} \Gamma$. 
Suppose now that $\hat{G}$ is core-free in $X$. Then $X$ has an exact factorization with core-free factors:

$$
X=\hat{G} H, \text { with } H, \hat{G} \text { core-free, and } \hat{G} \cap H=1,
$$

where $H=X_{\mathbb{1}}$. The action of $X$ on $[X: \hat{G}]$ gives rise to Cayley graphs of the group $H$, called dual Cayley graphs of $\Gamma$. In this case, we observe that the order of $X$ and hence the order of $G$ are up-bounded in terms of the order of $X_{\mathbb{1}}$; that is,

$$
\hat{G}<X \leq \operatorname{Sym}\left(\left|X_{\mathbb{1}}\right|\right) \text {. }
$$

It is known that for many important classes of graphs, the order of $X_{\mathbb{1}}$ is further upbounded in terms of the valency: for example, cubic edge-transitive graphs, 2-arc transitive graphs, and vertex-primitive graphs. This therefore gives an up-bound of $|G|$ and $|X|$ in terms of the valency. This implies that $X$ has two faithful transitive permutation representations, that is, on $[X: \hat{G}]$ and on $[X: H]$, and the statements in the following lemma are obviously true.

Lemma 3.1. Let $\Gamma$ be a Cayley graph of $G$, and let $X \leq$ Aut $\Gamma$ be such that $\hat{G}<X$ and $\operatorname{core}_{X}(\hat{G})=1$. Then $X$ has a faithful transitive permutation representation on the set $[X: \hat{G}]$, and $X_{\mathbb{1}}$ is a regular subgroup of $X$ acting on $[X: \hat{G}]$. In particular, the order $|X|$ is up-bounded in terms of $\left|X_{\mathbb{1}}\right|$.

Each generalized orbital graph of $X$ on the set $[X: \hat{G}]$ is therefore a Cayley graph of the group $H=X_{\mathbb{1}}$, which is a dual Cayley graph of Cay $(G, S)$. Considering the dual permutation representation of a vertex-primitive automorphism group of a Cayley graph, we have the following result.

Proposition 3.2. For every given integer $k$, there are only finitely many vertexprimitive Cayley graphs of valency $k$ that are not normal Cayley graphs.

Proof. Let $\Gamma=\operatorname{Cay}(G, S)$ be such that $X=\operatorname{Aut} \Gamma$ is primitive on $V \Gamma$. It follows that $\hat{G}$ in $X$ is either normal or core-free. By [5], the order of $X_{\mathbb{1}}$ is up-bounded in terms of the valency of an orbital graph of $X$ on $V \Gamma$, where $\mathbb{1}$ is the identity of $G$. Thus $\left|X_{\mathbb{1}}\right|$ is up-bounded in terms of the valency of $\Gamma$. If $\Gamma$ is core-free, then $X$ has a faithful representation on $[X: \hat{G}]$, which is of degree $\left|X_{\mathbb{1}}\right|$. Hence $|X| \leq\left|X_{\mathbb{1}}\right|$ !, and so the order of $X$ is up-bounded in terms of the valency of $\Gamma$, and so the number of vertex-primitive Cayley graphs of valency $k$ that are not normal Cayley graphs is up-bounded in terms of $k$.

This shows that, although they are very special, normal Cayley graphs are not very rare. Similarly, we have the next proposition regarding 2-arc transitive Cayley graphs.

Proposition 3.3. For any given positive integer $k$, there are at most finitely many core-free 2-arc transitive Cayley graphs of valency $k$.

Proof. Let $\Gamma=\operatorname{Cay}(G, S)$ be an $(X, 2)$-arc transitive graph of valency $k=|S|$ such that $\hat{G}<X \leq$ Aut $\Gamma$ and $\hat{G}$ is core-free in $X$. By [23], the order of $X_{\mathbb{1}}$ is up-bounded in terms of the valency $k$, where $\mathbb{1}$ is the identity of $G$. Since $\hat{G}$ is core-free in $X$, the group $X$ has a faithful transitive representation on $[X: \hat{G}]$, which is of degree $\left|X_{\mathbb{1}}\right|=|X: \hat{G}|$. Hence $|X| \leq\left|X_{\mathbb{1}}\right|$ !, and so the order of $X$ is up-bounded in terms of $k$. In particular, the number of core-free 2 -arc transitive Cayley graphs of valency $k$ is up-bounded in terms of $k$. 


\section{Examples}

In this section, we construct examples of $s$-arc transitive core-free Cayley graphs, where $s \geq 2$.

It is obvious that a complete graph $\mathrm{K}_{n}$ of $n$ vertices with $n \geq 4$ has automorphism group isomorphic to $S_{n}$ and is a 2-transitive Cayley graph of an arbitrary group of order $n$. Also, it is easy to see that a complete bipartite graph $\mathrm{K}_{n, n}$ with $n \geq 3$ has automorphism group $\mathrm{S}_{n}<\mathrm{S}_{2}$, and is a 3-transitive Cayley graph of valency $n$. This shows that $\mathcal{G}(2, k)$ and $\mathcal{G}(3, k)$ are nonempty for all $k \geq 3$. Except for these "trivial" examples, various other $s$-arc transitive core-free Cayley graphs will be constructed in this section.

Let $X$ be a group, and let $H$ be a core-free subgroup of $X$. Let $[X: H]=\{H x \mid$ $x \in X\}$. For an element $g \in X$ such that $g^{2} \in H$, a coset graph

$$
\Gamma:=\operatorname{Cos}(X, H, H g H)
$$

is defined as the graph with vertex set $V=[X: H]$ such that $H x$ is adjacent to $H y$ if and only if $y x^{-1} \in H g H$. We have the following well-known properties.

Lemma 4.1. Let $\Gamma=\operatorname{Cos}(X, H, H g H)$. Then

(i) $\Gamma$ is connected if and only if $\langle H, g\rangle=X$;

(ii) $\Gamma$ is $(X, 2)$-arc transitive if and only if $H$ is 2-transitive on $\left[H: H \cap H^{g}\right]$.

The first example gives a family of 2-arc transitive core-free Cayley graphs of prime-power valency.

Example 4.2. Let $X=\mathrm{S}_{p^{r}}$ where $p$ is a prime, acting on $\Omega=\left\{1,2, \ldots, p^{r}\right\}$. Then $X$ has two subgroups $G, H$ such that $G=X_{1,2} \cong \mathrm{S}_{p^{r}-2}$ and $H \cong \mathrm{AGL}_{1}\left(p^{r}\right) \cong$ $\mathbb{Z}_{p}^{r} \rtimes \mathbb{Z}_{p^{r}-1}$ is 2 -transitive on $\Omega$. It follows that $G \cap H=1$ and $X=G H$. Write $H=N \rtimes\langle z\rangle$, where $N \cong \mathbb{Z}_{p}^{r}$ is regular on $\Omega$, and $z=\left(1,2,3, \ldots, p^{r}-1\right)$. It is easily shown that there exists an involution $g \in X$ such that $z^{g}=z^{-1}$ and $\langle H, g\rangle=X$. Let $\Gamma=\operatorname{Cos}(X, H, H g H)$. Then $\Gamma$ is a connected 2-arc transitive graph of valency $p^{r}$ and is a Cayley graph of $G$.

The next two examples were constructed in [16] as examples of quasiprimitive 2-arc transitive graphs of a certain type. Here we prove they are Cayley graphs.

Example 4.3. Let $\Gamma$ be a graph constructed in Example 4.2 with $r=1$ and $p \geq 5$. Then $\Gamma$ is a 2-arc transitive Cayley graph of $S_{p-2}$. Let $l$ be a divisor of $(p-1) / 2$, and let

$$
Y=\left(T_{1} \times T_{2} \times \cdots \times T_{l}\right) \rtimes(O \times L),
$$

where $T_{i} \cong \mathrm{A}_{p}, O \cong \mathbb{Z}_{2}$ normalizes but does not centralize $T_{i}$ for each $i$, and $L=\langle(1,2,3, \ldots, l)\rangle \cong \mathbb{Z}_{l}$ permutes cyclically the direct factors $T_{1}, T_{2}, \ldots, T_{l}$.

It is shown in [16] that $Y$ has a subgroup $K \cong\left(\mathbb{Z}_{p} \rtimes \mathbb{Z}_{p-1}\right) \times \mathbb{Z}_{l}$ and an element $g \in Y$ such that the coset graph $\Gamma:=\operatorname{Cos}(Y, K, K g K)$ is connected and $(Y, 2)$-arc transitive of valency $p$. It is now easily shown that the subgroup $R \times T_{2} \times \cdots \times T_{l}$, where $\mathrm{S}_{p-2} \cong R<T_{1}$, acts regularly on $V \Gamma$. Hence $\Gamma$ is a Cayley graph.

The following example presents a family of 3-arc transitive Cayley graphs.

Example 4.4. Let $T=\operatorname{PSL}(2, q)$, where $q=2^{e}$ with $e \geq 2$, and let $H=$ $\mathbb{Z}_{2}^{e}: D$, where $D \cong \mathbb{Z}_{2^{e}-1}$. Let $f \in T$ be such that $\langle D, f\rangle \cong \mathrm{D}_{2\left(p^{e}-1\right)}$. Then $\Sigma=\operatorname{Cos}(T, H, H f H) \cong \mathrm{K}_{q+1}$ is $(T, 2)$-arc transitive. Let

$$
Y=\left(T_{1} \times T_{2} \times \cdots \times T_{q-1}\right) \rtimes L,
$$


where $T_{i} \cong \mathrm{A}_{p}$, and $L=\langle(1,2,3, \ldots, q-1)\rangle \cong \mathbb{Z}_{q-1}$ permutes cyclically the direct factors $T_{1}, T_{2}, \ldots, T_{q-1}$.

It is shown in [16] that $Y$ has a subgroup $K \cong\left(\mathbb{Z}_{p} \rtimes \mathbb{Z}_{q-1}\right) \times \mathbb{Z}_{q-1}$ and an element $g \in Y$ such that the coset graph $\Gamma:=\operatorname{Cos}(Y, K, K g K)$ is connected and $(Y, 3)$-arc transitive of valency $p$. It is now easily shown that the subgroup $R \times T_{2} \times \cdots \times T_{l}$, where $\mathbb{Z}_{q+1} \cong R<T_{1}$, acts regularly on $V \Gamma$. Hence $\Gamma$ is a Cayley graph.

Finally, we present a family of 4-arc transitive Cayley graphs.

Example 4.5. Let $\Gamma$ be the incidence graph of a Desarguesian projective plane $\Pi$ of order $n$. Then $n$ is a prime power, and $\operatorname{Aut} \Pi=\operatorname{PGL}(3, n)$; see [6]. It is known that the incidence graph $\Gamma$ is 4 -arc transitive of valency $n+1$, and $\operatorname{Aut} \Gamma=$ Aut $\Pi . \mathbb{Z}_{2}=\operatorname{Aut}(\operatorname{PSL}(3, n))$. Now the Singer subgroup $C \cong \mathbb{Z}_{n^{2}+n+1}$ is regular on both the point set $\mathcal{P}$ and the line set $\mathcal{L}$. There exists an involution $\tau \in \operatorname{Aut} \Gamma \backslash$ Aut $\Pi$ such that $\tau$ normalizes $C$ (refer to [10, Lemma 3.2]). Hence Aut $\Gamma$ has a subgroup $G:=\langle C, \tau\rangle \cong \mathbb{Z}_{n^{2}+n+1} \rtimes \mathbb{Z}_{2}$ that is regular on the vertices of the graph $\Gamma$. So $\Gamma$ is a Cayley graph of a group isomorphic to $G$.

\section{Proofs of Theorems 1.1 and 1.4}

In this section, we prove Theorems 1.1 and 1.4 .

Proof of Theorem 1.1. Let $\Gamma=\operatorname{Cay}(G, S)$ be an $(X, s)$-arc transitive graph of valency $k$ with vertex set $V$, where $s \geq 2$. Assume furthermore that $\hat{G}<X$. Let $C=\operatorname{core}_{X}(\hat{G})$.

Assume first that $C=\hat{G}$. Then $\hat{G}$ is normal in $X$, and hence by Proposition 2.3 . we have that $s=2$, as in part (i) of Theorem 1.1.

Assume that $C$ has exactly two orbits in $V$. Then $\hat{G}$ is bi-normal in $X$, and $\Gamma$ is bipartite. Hence by Corollary 2.7, we have that either $s=2$, or $(s, k)=(3,7)$, as in part (i) of Theorem 1.1

Finally, assume that $C$ has at least three orbits in $V$. Then $\hat{G} / C$ is core-free in $X / C$ and regular on the vertex set of $\Gamma_{C}, X / C \leq \operatorname{Aut}_{C}$, and $\Gamma_{C}$ is an $(X / C, s)$-arc transitive graph of valency $k$. Thus $\Gamma_{C}$ is a member of $\mathcal{G}(s, k)$, and $\Gamma$ is a normal cover of $\Gamma_{C}$, as in part (ii) of Theorem 1.1.

The existence of graphs in $\mathcal{G}(s, k)$ with $s \leq 4$ is justified by the examples given in Section 4. This completes the proof of Theorem 1.1.

To prove Theorem 1.4 we first investigate the graphs $\Gamma(q)$. Let $n$ be a prime power such that $p:=n^{2}+n+1$ and $q:=n+1$ are primes. Let

$$
G=\mathrm{D}_{2 p}=\langle a\rangle \rtimes\langle z\rangle,
$$

where $a^{z}=a^{-1}$. Let $\sigma \in \operatorname{Aut}(\langle a\rangle)$ be such that $\sigma z=z \sigma$ and $o(\sigma z)=2 q$. Let

$$
S=(a z)^{\langle\sigma\rangle}=\left\{a z, a^{r} z, a^{r^{2}} z, \ldots, a^{r^{q-1}} z\right\},
$$

and set $\Gamma(q)=\operatorname{Cay}(G, S)$.

Lemma 5.1. Let $\Gamma=\Gamma(q)$, and let $d(\Gamma)$ and $g(\Gamma)$ be the diameter and the girth of $\Gamma$, respectively. Then

(i) $\Gamma$ is of valency $q$, and Aut $\Gamma=\langle a\rangle \rtimes\langle z \sigma\rangle \cong \mathbb{Z}_{p} \rtimes \mathbb{Z}_{2 q}$;

(ii) either $(d(\Gamma), g(\Gamma))=(3,6)$, or $g(\Gamma)=4$. 
Proof. The graph $\Gamma$ is a bipartite graph of valency $q$ with biparts $\Delta$ and $\Delta^{\prime}$ say. Write $A=$ Aut $\Gamma$ and $A^{+}=A+\Delta=A_{\Delta^{\prime}}$. Then $A \geq G \rtimes\langle\sigma\rangle$, and $A^{+} \geq\langle a\rangle \rtimes\langle\sigma\rangle \cong$ $\mathbb{Z}_{p} \rtimes \mathbb{Z}_{q}$. In particular, $A^{+}$is a primitive permutation group of degree $p$. Thus by Burnside's theorem, either $A^{+}$is affine or an almost simple group. It then follows from the result of [9] that either $\langle a\rangle \triangleleft A^{+}$, or $\operatorname{soc}\left(A^{+}\right)=\operatorname{PSL}(d, r)$ with $p=\frac{r^{d}-1}{r-1}$, or $\mathrm{A}_{p}$. Suppose that $\operatorname{soc}\left(A^{+}\right)=\operatorname{PSL}(d, r)$. Then the cyclic group $\langle a\rangle$ is a Singer cycle of $\operatorname{PSL}(d, r)$, and so $\mathrm{N}_{\mathrm{PSL}(d, r)}(\langle a\rangle) \leq\langle a\rangle \rtimes \mathbb{Z}_{d}$. Thus the order $o(\sigma)$ divides $d$, which is not possible. Suppose that $\operatorname{soc}\left(A^{+}\right)=\mathrm{A}_{p}$. Then $A_{\mathbb{1}}=\mathrm{A}_{p-1}$. Since the smallest transitive permutation representation of $\mathrm{A}_{p-1}$ is $p-1$, it follows that $\Gamma$ has valency at least $p-1$, which is a contradiction. Thus $\langle a\rangle$ is normal in $A^{+}$. It then follows that Aut $\Gamma=G \rtimes\langle z \sigma\rangle$, as in part (i).

Suppose that the girth $g(\Gamma)$ of $\Gamma$ is at least 6 . Since $|\Gamma(\mathbb{1})|=n+1$, we have that $\left|\Gamma_{2}(\mathbb{1})\right|=n^{2}+n$. Furthermore, since $|V \Gamma|=2\left(n^{2}+n+1\right)$, we conclude that $\left|\Gamma_{3}(\mathbb{1})\right| \leq|V \Gamma|-\left(1+(n+1)+\left(n^{2}+n\right)\right)=n^{2}$. It then follows that $g(\Gamma)=6$, $\left|\Gamma_{3}(\mathbb{1})\right|=n^{2}$, and the diameter $d(\Gamma)=3$. Suppose finally that the girth $g(\Gamma)$ of $\Gamma$ is less than 6 . Then since $\Gamma$ is bipartite, $g(\Gamma)=4$.

Now we are ready to prove Theorem 1.4 .

Proof of Theorem 1.4 Let $\Pi$ be a projective plane of order $n$, where $n$ is a natural integer. Let $\Gamma$ be the incidence graph of $\Pi$; that is, the vertex set of $\Gamma$ is $\mathcal{P} \cup \mathcal{L}$, and the edge set of $\Gamma$ is the set of flags of $\Pi$. Then $\Gamma$ is a bipartite graph with biparts $\mathcal{P}$ and $\mathcal{L}$, and either $\operatorname{Aut} \Gamma=\operatorname{Aut} \Pi$, or $\operatorname{Aut} \Gamma=(\operatorname{Aut} \Pi) . \mathbb{Z}_{2}$.

Assume that $\Pi$ is not Desarguesian, and assume that $\Pi$ is flag-transitive. By 12 (or refer to [7), $n$ is a 2-power, both $q:=n+1$ and $p:=n^{2}+n+1$ are primes, and Aut $\Pi=\langle a\rangle \rtimes\langle\sigma\rangle \cong \mathbb{Z}_{p} \rtimes \mathbb{Z}_{q}$ is a Frobenius group, where $o(a)=p$ and $o(\sigma)=q$.

The subgroup Aut $\Pi$ of Aut $\Gamma$ acts transitively on the edge set of $\Gamma$, and furthermore, the cyclic subgroup $\langle a\rangle$ of Aut $\Pi$ is regular on each of the biparts $\mathcal{P}$ and $\mathcal{L}$. Thus by [10. Lemma 3.2], there exists an element $z \in A u t \Gamma$ interchanging points and lines of $\Pi$. It follows that $\operatorname{Aut} \Gamma=(\operatorname{Aut} \Pi) \cdot \mathbb{Z}_{2}=\langle a, \sigma, z\rangle \cong \mathbb{Z}_{p} \rtimes \mathbb{Z}_{2 q}$, and the subgroup $G:=\langle a, z\rangle$ is regular on the vertex set $\mathcal{P} \cap \mathcal{L}$. Thus $\Gamma$ may be viewed as a Cayley graph of $G$, say $\Gamma=\operatorname{Cay}(G, S)$ for some subset $S \subset G$.

Since $\Gamma$ is the incidence graph of the projective plane $\Pi, \Gamma$ has girth 6 and diameter 3. It follows that $G$ is not abelian and that Aut $\Gamma$ is a Frobenius group. In particular, $G$ is a dihedral group of order $2 p$.

Now $G$ is normal in Aut $\Gamma$, and hence $\Gamma$ is a normal Cayley graph of $G$. By Lemma 2.1, we have that $\operatorname{Aut} \Gamma=G \rtimes \operatorname{Aut}(G, S)$. Hence $(\operatorname{Aut} \Gamma)_{\mathbb{1}}=\operatorname{Aut}(G, S) \cong$ $\mathbb{Z}_{q}$. Since all subgroups of Aut $\Gamma$ of order $q$ are conjugate, we may assume that $\operatorname{Aut}(G, S)=\langle\sigma\rangle$. Since $\Gamma$ is bipartite, we conclude that $S \cap\langle a\rangle=\emptyset$, and so all elements of $S$ are involutions. Thus $a^{i} z \in S$ for some $i$ with $1 \leq i \leq p-1$. Let $a^{\sigma}=a^{r}$ for some integer $r$. Then $S=\left(a^{i} z\right)^{\langle\sigma\rangle}=\left\{a^{i} z, a^{r i} z, \ldots, a^{r^{q-1}} i z\right\}$, and so $\Gamma \cong \Gamma(q)$, as defined before.

Conversely, assume that $\Gamma(q)$ has girth 6 for some $q$. By Lemma 5.1 the diameter of $\Gamma(q)$ equals 3. Let $\mathcal{P}$ and $\mathcal{L}$ be the biparts of $\Gamma(q)$, and call the vertices in $\mathcal{P}$ points and the vertices in $\mathcal{L}$ lines. Then it is easily shown that the incidence structure $\Pi=(\mathcal{P}, \mathcal{L})$ is a projective plane of order $q-1$. By Lemma 5.1 we have $\operatorname{Aut} \Gamma(q) \cong \mathbb{Z}_{p} \rtimes \mathbb{Z}_{2 q}$. It is known that a Desarguesian projective plane of order $q-1$ has automorphism group $\operatorname{PGL}(3, q-1)$; see, for example, 6]. So $\Pi$ is not Desarguesian. 


\section{ADDED IN PROOF}

A remark on Question 1.2(b). A 5-arc transitive Cayley graph is constructed by $\mathrm{Xu}$, Fang, Wang and $\mathrm{Xu}$ in On cubic s-arc transitive Cayley graphs of finite simple groups, to appear in European Journal of Combinatorics. Also M. Conder announced that he has constructed some infinite families of $s$-arc transitive Cayley graphs for $s=5$ or 7 .

\section{REFERENCES}

[1] B. Alspach, M. Conder, D. Marusic and M. Y. Xu, A classification of 2-arc-transitive circulants, J. Algebraic Combin. 5 (1996), no. 2, 83-86. MR97a:05099

[2] R. Baddeley, Two-arc transitive graphs and twisted wreath products, J. Algebraic Combin. 2 (1993), 215-237. MR94h:05037

[3] N. Biggs, Algebraic Graph Theory, Cambridge University Press, 2nd edition, New York, 1992. MR95h:05105

[4] P. J. Cameron, Permutation groups, London Mathematical Society Student Texts, 45. Cambridge University Press, Cambridge, 1999. x+220 pp. MR2001c:20008

[5] P. J. Cameron, C. E. Praeger, J. Saxl and G. M. Seitz, On the Sims conjecture and distance transitive graphs, Bull. London Math. Soc. 15 (1983), 499-506. MR85g:20006

[6] P. Dembowski, Finite Geometries, Springer, New York, 1968. MR38:1597

[7] W. Feit, Finite projective planes and a question about primes, Proc. Amer. Math. Soc. 108 (1990), 561-564. MR90e:51016

[8] C. D. Godsil, On the full automorphism group of a graph, Combinatorica 1 (1981), 243-256. MR 83a:05066

[9] R. Guralnick, Subgroups of prime power index in a simple group, J. Algebra 81 (1983), 304-311. MF $84 \mathrm{~m}: 20007$

[10] A. A. Ivanov and M. E. Iofinova, Biprimitive cubic graphs, in Investigations in the Algebraic Theory of Combinatorial Objects, Math. and its Applications (Soviet Series), Vol. 84 (1993), 459-472, Kluwer, Dordrecht, Boston, London. MR88m:05048

[11] A. A. Ivanov and C. E. Praeger, On finite affine 2-arc transitive graphs, Europ. J. Combin. 14 (1993), 421-444. MR94k:05089

[12] W. M. Kantor, Primitive groups of odd degree and an application to finite projective planes, J. Algebra, 106 (1987), 15-45. MR88b:20007

[13] C. H. Li, Finite $s$-arc transitive graphs of prime-power order, Bull. London Math. Soc. 33 (2001), 129-137. MR.2002d:05064

[14] C. H. Li, The finite vertex-primitive and vertex-biprimitive $s$-transitive graphs with $s \geq 4$, Trans. Amer. Math. Soc. 353 (2001), 3511-3529. MR2002c:05084

[15] C. H. Li, On finite 2-arc-transitive Cayley graphs, submitted.

[16] C. H. Li and A. Seress, Finite quasiprimitive $s$-arc transitive graphs of product action type, in preparation.

[17] H. van Maldeghem, Generalized Polygons, Birkhäuser Verlag, Boston, Berlin, 1998. MR 2000k:51004

[18] D. Marusic, On 2-arc-transitivity of Cayley graphs, J. Combin. Theory Ser. B 87 (2003), no. 1, 162-196. MR.2004a:05064

[19] C. E. Praeger, An O'Nan-Scott theorem for finite quasiprimitive permutation groups and an application to 2-arc transitive graphs, J. London. Math. Soc. 47 (1992), 227-239. MR 94f:05068

[20] C. E. Praeger, On a reduction theorem for finite bipartite 2-arc transitive graphs, Australas. J. Combin. 7 (1993), 21-36. MR93m:05091

[21] C. E. Praeger, Finite normal edge-transitive Cayley graphs, Bull. Austral. Math. Soc. 60 (1999), 207-220. MR2000j:05057

[22] Koen Thas, Finite flag-transitive projective planes: a survey and some remarks, Discrete Math. 266 (2003), 417-429.

[23] V. I. Trofimov and R. M. Weiss, Graphs with a locally linear group of automorphisms, Math. Proc. Cambridge Philos. Soc. 118 (1995), 191-206. MR97d:05143 
[24] R. M. Weiss, The nonexistence of 8-transitive graphs, Combinatorica 1 (1981), 309-311. MR $84 f: 05050$

[25] M. Y. Xu, Automorphism groups and isomorphisms of Cayley digraphs, Discrete Math. 182 (1998), 309-320. MR 98i:05096

School of Mathematics and Statistics, The University of Western Australia, Crawley, 6009 Western Australia, Australia

E-mail address: li@maths.uwa.edu.au 